\title{
Effects of delivery via pressure-adjustable pneumatic gas- powered dart gun of three antimicrobial drugs (ceftiofur crystalline free acid, tildopirosin, and tulathromycin) on drug disposition and meat quality in cattle
}

\author{
Thomas B. Hairgrove ${ }^{1}$, Virginia Fajt ${ }^{\text {Corresp., } 2}{ }^{2}$, Ronald Gill ${ }^{1}$, Rhonda Miller ${ }^{1}$, Michael Miller ${ }^{1}$, Travis Mays $^{3}$ \\ ${ }^{1}$ Department of Animal Science, Texas A\&M University, College Station, TX, United States \\ 2 Department of Veterinary Physiology and Pharmacology, Texas A\&M University, College Station, TX, United States \\ 3 Texas Veterinary Medical Diagnostic Laboratory, College Station, TX, United States \\ Corresponding Author: Virginia Fajt \\ Email address: vfajt@cvm.tamu.edu
}

Background. Although Beef Quality Assurance guidelines do not recommend use of darting methods to deliver drugs, cattle in the US may be raised on farms and ranches without restraint facilities, and reports from the field suggest that dart guns are being used to deliver antimicrobial drugs. Few studies report whether this route of administration results in altered drug disposition or carcass quality. Methods. Forty steers were blocked by sire and then randomly assigned to treatment with saline, ceftiofur crystalline free acid, tildipirosin, or tulathromycin delivered via dart gun. To assess drug disposition, 8 ceftiofur, 6 tulathromycin, and 6 tildipirosin-treated calves were selected to measure plasma concentrations of drugs up to 10 days after drug administration. Steers were then fed a balanced ration for approximately 6.5 months and slaughtered. To evaluate carcass quality, tenderness of steaks from darted-side and non-darted sides was evaluated via Warner-Bratzler shear force testing. Due to the prohibition of extralabel routes of administration for ceftiofur in the U.S., animals treated with this drug did not enter the food supply. Results. Ceftiofur disposition differed from published reports with lower mean $\mathrm{C}_{\max }$ but similar mean apparent elimination half-life. Tildipirosin disposition differed from published reports with lower $\mathrm{C}_{\max }$ and shorter apparent elimination half-life.

Tulathromycin was similar to previous published reports but $\mathrm{C}_{\max }$ and apparent elimination half-life was highly variable. All steaks (from darted and non-darted sides) from cattle treated with ceftiofur and saline were more tender than from cattle treated with tulathromycin or tildipirosin $(P=0.003)$. There was a trend toward more tenderness in steaks from the non-darted compared to the darted side. Steaks from the darted side for one treatment, tildipirosin, were less tender than the non-darted side. Conclusions. 
Pharmacokinetic parameters of ceftiofur crystalline free acid, tildipirosin, and tulathromycin to cattle using pressure-adjustable pneumatic gas-powered dart gun were estimated in this study. Delivery of tildipirosin and tulathromycin to cattle with dart gun may also result in detectable decreases in tenderness of harvested steaks. 
1 Title

2 Effects of delivery via pressure-adjustable pneumatic gas-powered dart gun of three

3 antimicrobial drugs (ceftiofur crystalline free acid, tildopirosin, and tulathromycin) on

4 drug disposition and meat quality in cattle

5

6 Tom Hairgrove ${ }^{1}$, Virginia R. Fajt ${ }^{2}$, Ronald Gill ${ }^{1}$, Rhonda Miller ${ }^{1}$, Michael Miller ${ }^{*}$, and Travis

7 Mays $^{3}$

8

$9 \quad{ }^{1}$ Department of Animal Science, College of Agriculture and Life Science, Texas A\&M

10 University, College Station, TX, US

$11{ }^{2}$ Veterinary Physiology and Pharmacology, College of Veterinary Medicine and Biomedical

12 Sciences, Texas A\&M University, College Station, TX, US

$13{ }^{3}$ Texas Veterinary Diagnostic Laboratory, College Station, TX, US

14

*Current affiliation: W.H. Miner Institute, Chazy, NY, US

16

17 Corresponding author: Virginia Fajt

18 vfajt@cvm.tamu.edu

19

20 
21 Abstract

Background. Although Beef Quality Assurance guidelines do not recommend use of darting methods to deliver drugs, cattle in the US may be raised on farms and ranches without restraint facilities, and reports from the field suggest that dart guns are being used to deliver antimicrobial drugs. Few studies report whether this route of administration results in altered drug disposition or carcass quality.

Methods. Forty steers were blocked by sire and then randomly assigned to treatment with saline, ceftiofur crystalline free acid, tildipirosin, or tulathromycin delivered via dart gun. To assess drug disposition, 8 ceftiofur, 6 tulathromycin, and 6 tildipirosin-treated calves were selected to measure plasma concentrations of drugs up to 10 days after drug administration. Steers were then fed a balanced ration for approximately 6.5 months and slaughtered. To evaluate carcass quality, tenderness of steaks from darted-side and non-darted sides was evaluated via Warner-Bratzler shear force testing. Due to the prohibition of extralabel routes of administration for ceftiofur in the U.S., animals treated with this drug did not enter the food supply.

Results. Ceftiofur disposition differed from published reports with lower mean $\mathrm{C}_{\max }$ but similar mean apparent elimination half-life. Tildipirosin disposition differed from published reports with lower $\mathrm{C}_{\max }$ and shorter apparent elimination half-life. Tulathromycin was similar to previous published reports but $\mathrm{C}_{\max }$ and apparent elimination half-life was highly variable. All steaks (from darted and non-darted sides) from cattle treated with ceftiofur and saline were more tender than from cattle treated with tulathromycin or tildipirosin $(\mathrm{P}=0.003)$. There was a trend toward more tenderness in steaks from the non-darted compared to the darted side. Steaks from the darted side for one treatment, tildipirosin, were less tender than the non-darted side. 
44 Conclusions. Pharmacokinetic parameters of ceftiofur crystalline free acid, tildipirosin, and

45 tulathromycin to cattle using pressure-adjustable pneumatic gas-powered dart gun were

46 estimated in this study. Delivery of tildipirosin and tulathromycin to cattle with dart gun may

47 also result in detectable decreases in tenderness of harvested steaks. 
48

49

50

51

52

53

54

55

56

57

58

59

60

61

62

63

64

\section{Introduction}

Stocker cattle are recently weaned calves that are placed on pasture and then allowed to grow to approximately $300 \mathrm{~kg}$ before being placed in a feedyard for the final phase of feeding prior to slaughter (Falconer \& Anderson 2006). Texas has historically been a large producer of stocker cattle, with Texas A\&M AgriLife economists estimating 3.4 million stocker cattle in 2014 (personal communication, S. Bevers, 2018). Stocker cattle enterprises are more short-term than other phases of beef cattle production, with many producers not consistently engaged from year to year. Most antimicrobials used in stocker operations are prescription drugs and require veterinary oversight; however, anecdotal reports from stocker operators and agricultural extension personnel indicate that there is inadequate understanding of "extralabel use" and that drugs are commonly used in an unapproved and sometimes illegal manner. The National Cattlemen's Beef Association has stated that: "The companies manufacturing, selling and promoting these methods of drug and product delivery have the responsibility and the obligation to develop data to establish efficacy, safety, animal welfare, food safety, and other concerns as compared to current BQA [Beef Quality Assurance] approved methods of drug/product administration. It is also possible that FDA approval may be required for drug delivery by these methods of injecting drugs/products and that issue needs to be addressed by the manufacturers. Until such time as this critical data becomes available these methods do not meet BQA injectable product administration guidelines." However, anecdotal evidence suggests that darts are being used regularly, given that most stocker cattle are on pastures without working or capture facilities to use when treating cattle with injectable drugs. Given the few reports in the literature on the disposition of drugs administered via darts, the first objective of this study was to determine if the pharmacokinetics of three commonly used antibiotics are altered by using this 
71 route of administration. The antimicrobial products used in this study are single-dose injectable

72 products approved for the treatment of pneumonia in cattle.

74 In addition to changes in drug disposition, previous studies have shown that injections lead to

75 damage to the injected area, with the resultant remodeling and fibrous infiltrate leading to

76 changes in muscle tenderness (George et al. 1996; George et al. 1995; Sullivan et al. 2009).

77 Tenderness is an important contributor to consumer satisfaction of whole meat products such as

78 steaks. Its importance is underlined by beef quality assurance programs that urge that all

79 injectable products be administered subcutaneously rather than intramuscularly, when permitted

80 on the drug label. The second objective of this study was to evaluate injection site reactions

81 shortly after darting (13 days) and at the time of slaughter to determine if darting results in

82 changes in meat quality and tenderness.

84 Materials and Methods

85 Animals and drug administration

87 Forty steers were available from a project in which sires were genetically verified. Forty Bos 88 taurus-Bos indicus crossbred steers were purchased from the Department of Animal Science at

89 Texas A\&M University. Study design was a randomized block, with sires serving as blocks. The

90 steers belonging to each sire were randomly assigned to one of four treatment groups, 10 per

91 group. There were ten sires among the 40 animals, with seven sires represented across all four

92 treatment groups and three sires that were balanced across treatment groups as much as possible.

93 Treatment groups were (1) $6.6 \mathrm{mg} / \mathrm{kg}$ ceftiofur crystalline free acid (CCFA, Excede, Zoetis 
94 Animal Health, Kalamazoo, Michigan), (2) $4 \mathrm{mg} / \mathrm{kg}$ tildipirosin (TILD, Zuprevo, Merck Animal

95 Health, Madison, NJ), (3) $2.5 \mathrm{mg} / \mathrm{kg}$ tulathromycin (TULA, Draxxin, Zoetis Animal Health,

96 Kalamazoo, Michigan), and (4) $0.9 \%$ saline (SALN). Drugs were delivered by pressure

97 adjustable pneumatic gas-powered dart gun (X-Caliper compressed air rifle, Pneu-dart, Inc.

15223 Route 87 Highway Williamsport, PA 17701). Based on the manufacturer's

recommendations, a pressure of 6.9 bars (100 psi) was used for all treatments except CCFA.

Based on trial and error, CCFA was delivered using a pressure of 7.2 bars (104 psi) due to the

weight of the dart slowing the velocity enough to prevent full penetration of the skin at the

pressure used for the other drugs. A 16 gauge 1/2 inch single-port needle was used for all

treatments, and animals were darted at a distance of 25 feet aiming for a region approximately 4"

below the tuber ischii in order to target the biceps femoris muscle (also known as the bottom

round). Although Beef Quality Assurance guidelines recommend all injections be in the neck

whenever possible, the location of injection for this study was selected based on anecdotal

reports of how darts were being used in stocker cattle. In addition, it should be noted that the

administration of CCFA in this manner would be considered illegal, since cephalosporins may

not be administered via an extralabel route in the US (United States Food and Drug

subcutaneously at the base of or the middle posterior of the ear (United States Food and Drug be determined when animals are darted, so some drug may be subcutaneous and some may be intramuscular. All darts appeared to completely expel their contents, but darts were not weighed before or after administration. Darts were collected after administration, and no needles appeared 116 to remain in any animals. 
118 Five ceftiofur-, 6 tulathromycin-, and 6 tildipirosin-treated calves were randomly selected from 119 each group of 10 animals for sampling for plasma concentrations of drug. Three additional steers

120 from the herd (in addition from the 40 described above) were administered ceftiofur as

121 previously described at a separate time for pharmacokinetic assessments only to provide a better

122 estimate of pharmacokinetic parameters and verify the marked variability in drug concentrations;

123 no carcass quality or tenderness data were collected from these animals. Blood samples for drug

124 analysis were collected at 0, 4, 8 hours, and 1, 2, 3, 6 and 10 days after drug administration.

125 Animal availability and financial considerations prevented the inclusion of animals injected via 126 the conventional route of administration via syringe.

127

128 After treatment and blood collection, steers were assigned to one of three feedlot pens for 129 feeding so that sire and treatment groups were balanced across pens. Steers were fed a balanced 130 ration that included dry rolled corn, dried distillers' grains, chopped alfalfa, molasses, and a 131 mineral premix including lasalocid (1320 g/ton, resulting in $33 \mathrm{~g} /$ ton on a dry matter basis or 30 $132 \mathrm{~g} /$ ton at $90 \%$ dry matter as specified on the product label) for approximately 195 days $(6.5$ 133 months). Steers were then slaughtered with captive bolt and exsanguination.

135 All animal procedures were approved by the Texas A\&M University Institutional Animal Care 136 and Use Committee (IACUC \#2014-0316).

137

138 Drug analysis 
139 All chemicals and reagents were obtained from VWR Scientific, Randor PA USA and ACS

140 grade unless otherwise noted. D9-clenbuterol (C570000) and tulathromycin (T897150) were

141 obtained from Toronto Research Chemicals, Toronto Canada. Tildipirosin (sc-397306) was

142 obtained from Santa Cruz Biotechnology. Ceftiofur (32442) was obtained from Sigma Aldrich.

143 St. Louis IL USA. Reagent grade water (RG-water) was provided by in-house PureLab ULTRA

144 system, ELGA Water Technologies LLC, Lowell MA USA.

146 For sample preparation, $1 \mathrm{~mL}$ serum or plasma was aliquoted for analysis. Standard curves for the 147 analytes of interest were prepared by fortifying the sample aliquots prior to Solid Phase

148 Extraction (SPE) at concentrations of $0.5 \mathrm{ppb}$ to $1,000 \mathrm{ppb}$. D9-clenbuterol $(40 \mu \mathrm{L})$ was added to

149 all samples, calibrators and controls as an internal standard to improve the precision of the 150 quantitative analysis. Tildipirosin and tulathromycin were analyzed by High Performance Liquid

151 Chromatography Tandem Mass Spectrometry (LCMSMS) after isolation by SPE. SPE was 152 processed using SPEWare CEREX48 (SPEWare Corp, Baldwin Park CA USA) processor and 153 concentrator. $3 \mathrm{~mL} / 35 \mathrm{mg}$ WWP SPE columns (SPEWare \#676-0353) were used to extract the analytes of interest. SPE columns were preconditioned with $1 \mathrm{~mL}$ methanol, and $1 \mathrm{~mL}$ RG-water before application sample. Columns were washed with $1 \mathrm{~mL} \mathrm{RG-water} \mathrm{prior} \mathrm{to} \mathrm{drying} 10$ minutes with nitrogen at $45^{\circ} \mathrm{C}$. SPE columns were eluted with $1 \mathrm{~mL}$ methanol before evaporation 157 under nitrogen for 10 minutes at $45^{\circ} \mathrm{C}$. Residues were reconstituted with $100 \mu \mathrm{L} 5 \%$ acetonitrile 158 in RG-water prior to analysis by LCMSMS.

160 Ceftiofur and metabolites were analyzed after conversion to Desfuroylceftiofur Acetamide 161 (DFCA). The residue from sample extract (from WWP) was added $450 \mu \mathrm{L} 4 \% \mathrm{w} / \mathrm{v}$ 
162 Dithioerythritol in borate buffer $\mathrm{pH} 9$ (4.75 g disodium tetraborate, and $925 \mathrm{mg}$ potassium

163 chloride in $250 \mathrm{~mL}$ RG-water). Samples were capped and heated at $50^{\circ} \mathrm{C}$ for $15 \mathrm{~min} .200 \mu \mathrm{L}$

164 Iodoacetamide solution $14 \% \mathrm{w} / \mathrm{v}$ in $\mathrm{PO}_{4}$ buffer $(0.025 \mathrm{M}, \mathrm{pH} 7)$ was added to the vials and then

165 heated at $50^{\circ} \mathrm{C}$ for an additional $15 \mathrm{~min}$. Samples were cooled and $500 \mu \mathrm{L} 1 \mathrm{~N}$ acetic acid was

166 added prior to SPE. $3 \mathrm{~mL} / 35 \mathrm{mg}$ Trace-B (SPEWare \# 711-335M) were used to extract the DFC

167 using the equipment mentioned above. Columns were preconditioned with $1 \mathrm{~mL}$ methanol, $1 \mathrm{~mL}$

168 RG-water, and $500 \mu \mathrm{L} 1 \mathrm{~N}$ acetic acid. After sample application, columns were washed with 1

$169 \mathrm{~mL}$ RG-water, $500 \mu \mathrm{L} 1 \mathrm{~N}$ acetic acid and $1 \mathrm{~mL}$ methanol. Columns were then dried under

170 nitrogen at $45^{\circ} \mathrm{C}$ prior to elution with $2 \%$ ammonium hydroxide in methanol. Eluents were dried

171 under nitrogen at $45^{\circ} \mathrm{C}$. Residues were reconstituted with $100 \mu \mathrm{L} \mathrm{5 \%}$ acetonitrile in RG-water

172 prior to analysis by LCMSMS.

173

174 High Performance Liquid Chromatography tandem Mass Spectrometry was performed on a TSQ 175 Endura, Thermo Instruments, San Jose CA USA and Agilent 6410 Triple Quadrupole LC/MS. 176 Analytes were separated on an Acentis Express C18 2.1 x 100 mm HPLC column (\#53823-U, 177 Supelco Inc. Bellefonte PA USA) using a mobile phase of $0.1 \%$ formic acid in both RG-water 178 (solvent A) and LC-MS grade acetonitrile (solvent B). A solvent gradient was applied to the 179 column at a flow of $300 \mu \mathrm{L} / \mathrm{min}$ of $5 \%$ B to $95 \%$ B over 8 minutes. The LCMSMS was run in 180 electrospray mode with a 0.7 mass resolution on both quadrupoles.

$\underline{\text { Pharmacokinetic analysis }}$

183 Noncompartmental analysis was performed to estimate the pharmacokinetic parameters in 184 plasma for each individual animal for each drug (see Tables 1-3 for parameters). 
$\underline{\text { Post-mortem carcass quality and tenderness assays }}$

187 Steers were humanely slaughtered at the Rosenthal Meat Science and Technology Center, Texas 188 A\&M University, College Station, TX. Carcasses were chilled for 48 hours postmortem and the

Bicep femoris from the right and left sides was removed for further evaluation. The Longissimus dorsi lumborum was also removed from one side of each carcass. Products from animals treated with CCFA did not enter the food supply.

Live Weight of the animal just prior to slaughter was measured. Dressing percentage was defined as hot carcass weight divided by live weight times 100 , where hot carcass weight was determined prior to chilling. Carcasses were ribbed at the $12^{\text {th }}$ and $13^{\text {th }}$ rib interface. Marbling score, ribeye area $\left(\mathrm{cm}^{2}\right)$, kidney, pelvic and heart fat $(\%)$, and subcutaneous fat thickness $(\mathrm{mm})$ were determined (USDA 2017). Yield grade and quality were calculated (USDA 2017).

After USDA grade assessment, color CIE (International Commission of Light) L* (lightness; $100=$ white and $0=$ black), $a^{*}\left(\right.$ overall hue; $100=$ red and $0=$ green), and $b^{*}$ (overall hue; $100=$ yellow and $0=$ blue) color space values were assessed (Minolta Colorimeter, CR-300, $8 \mathrm{~mm}$ diameter head, $10^{\circ}$ standard observer, D65 light source; Minolta Co., Ramsey, NJ). White and black tiles were used daily to calibrate the Minolta Colorimeter. At the $12^{\text {th }}$ rib Longissimus dorsi interface, three values were obtained per carcass and averaged. Three random $\mathrm{pH}$ values were obtained from the same location using a puncture $\mathrm{pH}$ probe (Hach, H100, Loveland, CO) and averaged. This value was defined as the final carcass $\mathrm{pH}$. The $\mathrm{pH}$ meter was calibrated $(\mathrm{pH}$ 4.0 and $\mathrm{pH} 7.0$ standard buffers) at the start of each day. Color and $\mathrm{pH}$ were determined on the 
208 same lean surface as used for grade assessment. Hump height measurement of the Rhomboideus

209 muscle (hump) was taken at its highest point.

210

211 The presumed site of dart entry was identified via visual examination for increased connective

212 tissue or marbling or by digital palpation for slightly thicker, denser areas of muscle by a trained

213 veterinarian with no knowledge of treatment. From the darted side in the bottom round muscle,

214 five-2.54-cm bottom round steaks were cut, the first steak included the presumed site of the dart

215 entry (Position 1), and then 2 proximal (Position 2 and 3) and 2 distal (Position 4 and 5) $2.54 \mathrm{~cm}$

216 steaks were removed from the presumed site of dart entry. One corresponding $2.54 \mathrm{~cm}$ steak

217 from the control side (Position 6) was also collected in the similar anatomical location from the

218 dart side.

219

220 From the Longissimus dorsi lumborum muscle, beginning at the anterior edge, four-2.54 $\mathrm{cm}$ top

221 loin steaks were cut, vacuum-packaged and randomly assigned to aging for 1, 7, 14, or 21 days at

$2224^{\circ} \mathrm{C}$ (labeled as Positions 1-4 in Table 4). Bottom round steaks were vacuum-packaged and aged

223 for 14 days. For Warner-Bratzler shear force determination, top loin and bottom round steaks

224 were weighed, iron constantan thermocouples (TT-J-36-SLE, Omega Engineering, Inc.,

225 Stanford, CT) were inserted in the geometric center of each steak, and steaks were cooked on flat

226 top electric skillets (Hamilton Beach grill, Hamilton Beach/ Proctor-Silex, Inc., Southern Pines,

227 NC) to a final internal temperature of $70^{\circ} \mathrm{C}$. Internal cook temperature was monitored with hand-

228 held recorders (model HH-21, Omega Engineering, Inc., Stanford, CT). Steaks were turned after

229 reaching $35^{\circ} \mathrm{C}$ internal temperature. After cooking, cooked weight was recorded and cook yield

230 was calculated (Cook Yield $=100-(($ Cooked Chop Weight/ Raw Chop Weight $) * 100)$. 
232 Cooked steaks were chilled for $24 \mathrm{hrs}$ and then one $1.27 \mathrm{~cm}$ diameter core, parallel to the

233 longitudinal orientation of the muscle fibers, was removed from six-seven positions designated

234 on each steak. Steaks were assigned codes prior to cooking and laboratory personnel were

235 blinded to treaments. Cores were sheared once with Warner-Bratzler shearing device (United

236 Smart-1 Test System SSTM-500, United Calibration Corp., Huntington Beach, CA; 200 kg load

237 cell, $200 \mathrm{~mm} / \mathrm{minute}$ head speed) certified by United Testing Systems, Inc. using a Warner-

238 Bratzler stainless steel blade (1.168 cm thick). Mis-shaped cores were not used. The maximum

239 force in $\mathrm{kg}$ for cores from one steak were averaged.

241 Data analysis

242 Carcass grade data, color, $\mathrm{pH}$ and Warner-Bratzler shear force measures were analyzed using

243 Analysis of Variance with the Proc GLM procedure of SAS (v9.4, SAS Institute, Cary, NC) with

244 an alpha of $<0.05$. For grade data, color and $\mathrm{pH}$, carcass was defined as the experimental unit.

245 The dart treatment was defined as a main effect and the pen cattle were fed in was defined as a

246 fixed effect. Warner-Bratzler shear force data were analyzed for top loin steaks. Main effects

247 were defined as dart treatment, steak position, aging day and their subsequent two-way

248 interactions and pen fed was used as a fixed effect. For bottom round steaks, data were analyzed

249 with dart treatment and position of steak from the injection site, and their interaction as a main

250 effects. The pen cattle were fed in was also defined as a fixed effect. A third analysis for bottom

251 round steaks compared sides within a carcass for Warner-Bratzler shear force. The undarted side

252 was defined as a control and the darted side was defined as the treatment. For all analyses, least 
253 squares means were calculated. Differences in least squares means were determined using the

254 Tukey-Kramer function within SAS for multiple mean comparisons.

255

256 Results

257 Drug Concentrations and Pharmacokinetics

258 Mean drug concentrations at sampling time points for the 3 drugs are shown in Figures 1-3, and 259 mean and median pharmacokinetic estimates are in Tables 1-3.

261 Carcass Quality and Tenderness

262 Carcass quality and yield factors by dart treatment indicated that dressing percentage and $L^{*}$ 263 values were significantly different $(\mathrm{P}=0.05$ and $\mathrm{P}<0.001$, respectively) across treatment groups 264 (Table 5). Dressing percentage was lower $(\mathrm{P}=0.05)$ in cattle treated with SALN than cattle 265 treated with CCFA and TILD. CIE color space values indicated $L^{*}$ values were significantly 266 higher $(\mathrm{P}<0.001)$ in cattle treated with CCFA, TILD and TULA resulting in lighter lean color 267 than the cattle treated with the SALN control. Dart treatments had no effect on other carcass 268 quality and yield grade factors.

As expected, top loin steak Warner-Bratzler shear force values across all treatments and 271 positions were lower $(\mathrm{P}<0.001)$ at $14 \mathrm{~d}$ and $21 \mathrm{~d}$ age postmortem than at $1 \mathrm{~d}$ and $7 \mathrm{~d}$ aging times 272 (Table 4). Shear force values were also lower $(\mathrm{P}<0.001)$ in steaks aged $7 \mathrm{~d}$ when compared to 273 steaks $1 \mathrm{~d}$ postmortem. Neither the dart treatments $(\mathrm{P}=0.21)$ nor position within the strip loin $274(\mathrm{P}=0.73)$ affected Warner-Bratzler shear force values. 
276 There was no difference $(\mathrm{P}>0.05)$ among core sample tenderness within steaks. There was also

277 no difference in tenderness between steaks collected at the various positions within the bottom

278 rounds (Table 4). All steaks (from darted and non-darted sides) from cattle treated with ceftiofur

279 and saline were more tender than from cattle treated with tulathromycin or tildipirosin $(\mathrm{P}=0.003)$.

280 There was a trend $(\mathrm{P}=0.08)$ toward more tenderness in steaks from the non-darted compared to

281 the darted side (Table 4). Steaks from the darted side for one treatment, tildipirosin, were less

282 tender than the non-darted side $(\mathrm{P}<0.05)$.

283

284 Discussion

285 Drug Concentrations and Pharmacokinetics

286 It is important to acknowledge that a limitation of this study is the lack of direct comparisons to

287 conventionally injected antimicrobial drugs. Cattle availability and financial constraints

288 prevented us from including additional animals in our study, and future studies could provide

289 controlled comparisons of drug disposition after different routes or methods of administration.

290 The following discussion reviews comparisons to previously published estimates of the

291 pharmacokinetics of the 3 drugs after conventional subcutaneous injections. These comparisons

292 are acknowledged to be subjective in nature, and pharmacokinetic parameters can be variable

293 across studies and across individual animals.

294

Ceftiofur Crystalline Free Acid. In this discussion, ceftiofur includes the combination of parent

drug and its derivates, mainly desfuroylceftiofur or desfuroylceftiofuracetamide;

297

desfuroylceftiofur is microbiologically as active as parent drug against cattle pneumonia

298

pathogens, and desfuroylceftiofuracetamide is derived during sample processing from 
299 desfuroylceftiofur. Concentrations of ceftiofur reached a much lower $\mathrm{C}_{\max }$ than previously

300 reported after conventional injection in Holstein calves (4260 ng/ml in plasma) (Foster et al.

301 2016), in dairy cows (4150 ng/ml in serum) (Witte et al. 2011), in beef calves (6020 $\mathrm{ng} / \mathrm{ml} \mathrm{in}$

302 plasma) (Washburn et al. 2005), and in neonatal Holstein calves (3230 ng/ml) (Woodrow et al.

303 2016). This is in sharp contrast to the mean $C_{\max }$ in the present study of $127 \mathrm{ng} / \mathrm{ml}$ (range 16-238

$304 \mathrm{ng} / \mathrm{ml})$.

305

306 On the other hand, the median observed $\mathrm{T}_{\max }$ of 8 hours was similar to previous reports: $12 \mathrm{hrs}$

307 (median) (Woodrow et al. 2016), $13.1 \mathrm{hrs}$ (mean) (Foster et al. 2016), $24 \mathrm{hrs}$ (Witte et al. 2011),

308 although in the last study, samples were only collected at 2 and $24 \mathrm{hr}$, and $18 \mathrm{hr}$ (back-

309 transformed least squares means) (Washburn et al. 2005). This suggests that ceftiofur is being

310 absorbed at a similar rate after darting but not to a similar degree as injecting in the ear, the route

311 of administration indicated on the label. It is also important to reiterate that darting CCFA

312 products is illegal in the US, because cephalosporin use at an extralabel regimen in cattle is

313 prohibited (United States Food and Drug Administration Center for Veterinary Medicine 2012).

Mean elimination half-life (74 hours, range $=31-136$ hours) was in the range of previously

reported times of 61 (Woodrow et al. 2016), 62 (Washburn et al. 2005), and $104 \mathrm{hrs} \mathrm{(Foster} \mathrm{et} \mathrm{al.}$

317 2016). This suggests that the most important difference between darting and conventional

318 injection is the amount of drug that enters the animal, although the range of estimated half-lives

319 in this study might result in significant variability in estimates of meat withdrawal times via this

320 route of administration. Tissues were not tested for ceftiofur concentrations in this study,

321 however, so this is only speculation. 
323 It should also be noted that, regardless of potential issues with therapeutic effectiveness and

324 impact on meat withdrawal time, administering CCFA at any other site than the base of or

325 middle posterior portion of the ear in a subcutaneous manner would be considered illegal in the

326 US because of restrictions on extralabel use of cephalosporins (United States Food and Drug

327 Administration Center for Veterinary Medicine 2012).

328

Tildopirosin. Only one previous report of the pharmacokinetics of tildopirosin in cattle has been 330 published (Menge et al. 2012). Mean $\mathrm{C}_{\max }$ was $711 \mathrm{ng} / \mathrm{ml}$, and mean elimination half-life was

331210 hours. These values are quite different from the present study: mean $\mathrm{C}_{\max }$ was $368 \mathrm{ng} / \mathrm{ml}$,

332 mean elimination half-life was 109 hours (see Table 3). Several factors could help explain the

333 differences: blood sampling was performed more intensely early in the dosing period in the

334 previous study $(0.5,1,2$ hours $)$ with the $C_{\max }$ being reported at a mean $T_{\max }$ of $0.7 \mathrm{hr}$ compared

335 to the present study (first sample at 4 hours). It is quite possible that a higher $\mathrm{C}_{\max }$ would have

336 been observed if we had collected earlier samples. In the previous study, samples were collected

337 up to 21 days after drug administration, whereas we only collected for 10 days. Without access to

338 the raw data of the previous data, we cannot know for sure, but visual examination of the graph

339 of plasma drug concentrations over time suggests that the slope of the elimination curve is not

340 considerably different from 2-10 days as compared to 10-21 days. Since this slope is the main

341 contributor to the estimate of elimination half-life, we speculate that our estimates would not

342 have changed significantly if we had collected samples for longer, but we cannot know for sure.

343 A higher than desirable percent of AUC was extrapolated to infinity in the present study, that is,

$34424 \%$ in one animal and $56 \%$ in another. In all other animals $(n=4)$, average percent of AUC 
345 extrapolated to infinity was $10 \%$, suggesting that blood samples were collected for a sufficient

346 amount of time in those animals to adequately characterize pharmacokinetic values. These data

347 do suggest, however, that darting of tildopirosin could affect the disposition of the drug in cattle.

349 Tulathromycin. Mean $\mathrm{C}_{\max }(619 \mathrm{ng} / \mathrm{ml})$ in the present study was in the range of previous reports 350 of conventionally injected tulathromycin: $277 \mathrm{ng} / \mathrm{ml}$ (Cox et al. 2010), $489 \mathrm{ng} / \mathrm{ml}$ (Nowakowski 351 et al. 2004), and $414 \mathrm{ng} / \mathrm{ml}$ (Nowakowski et al. 2004), and $1820 \mathrm{ng} / \mathrm{ml}$ (Foster et al. 2016).

352 Perhaps the most relevant comparison is to Holstein calves administered tulathromycin via 353 pneumatic dart, in which the mean $C_{\max }$ was $206 \mathrm{ng} / \mathrm{ml}$ (Coetzee et al. 2018). It should be noted,

354 however, that in that study, there was not a significant difference between $\mathrm{C}_{\max }$ after

355 conventional injection and successful dart delivery of tulathromycin (Coetzee et al. 2018),

356 largely due to the high variability in individual $\mathrm{C}_{\max }$, but there was a difference in $\mathrm{C}_{\max }$ between

357 successful and unsuccessful darting. In the present study, we did not appreciate any failure in

358 dart delivery of drug, but mean $\mathrm{C}_{\max }$ observed in the present study suggests that darting may

359 result in enough variability in drug concentrations to alter estimates of meat withdrawal times.

Estimated apparent elimination half-life in the present study $(107 \mathrm{hrs})$ is similar to the previous

362

363

364

365

366

367

report of $111 \mathrm{hrs}$ in darted cattle (Coetzee et al. 2018). This estimate is somewhat longer than reported elimination half-life after conventionally injected tulathromycin in other studies in cattle (92 (Nowakowski et al. 2004), 81 (Foster et al. 2016), and 64 hrs (Cox et al. 2010)). However, sampling times were not as prolonged in our study as in two of the previous studies. One collected samples for 336 hrs (Nowakowski et al. 2004) and the other 360 hrs (Cox et al. 2010), which could have impacted the estimation of elimination half-life. 
369 Carcass Quality and Tenderness

370 We are not aware of previous reports on the effects of remote delivery devices on tenderness at

371 harvest, although a recent study of pneumatic darts similar to those used in the present study

372 evaluated muscle damage by quantification of serum creatine kinase up to 6 days after injection

373 of tulathromycin via dart (Coetzee et al. 2018). In that study, no difference was detected in

374 creatine kinase concentrations between dart-injection and hand-injection of tulathromycin, but

375 sham-injected animals were not included as a comparison in the study. In other studies, gross

376 lesions have been reported at injection sites after intramuscular injection, including two reports

377 of remote delivery devices that differ from the one used in the present study. Investigators in

378 Canada used a crossbow to deliver oxytetracycline and tilmicosin to the neck or round (exact

379 location not specified) region in cattle 30 days from slaughter, and at slaughter they noted lesions

380 in darted and hand-injected cattle, including granulation, neovascularization, and abscesses (Van

381 Donkersgoed 1999). In the other darting study, the drug was delivered in patented devices called

382 biobullets, which were designed to dissolve once delivered into the muscle (Edwards 1993). In

383 that study, some of the biobullets did not enter the skin properly, and the ones that did enter the

384 skin caused significant tissue damage when the animals were necropsied 2 hours after injection.

385 In the present study, we did not evaluate tissue damage in the early days after injection except

386 during gross examination of the pilot study animals, so it is unknown if muscle was damaged at

387 dart delivery during the study. However, the only statistically significant differences at slaughter

388 in tenderness as evaluated via the Warner-Bratzler method (see Table 4) were in bottom round

389 steaks from tildipirosin- and tulathromycin-darted animals as compared to saline- and ceftiofur-

390 darted animals. Consumers can detect differences of $0.5 \mathrm{~kg}$ in Warner-Bratzler tenderness 
391 measures (Miller, 2001), so the statistically significant differences noted between tildipirosin-

392 darted and non-darted steaks (Table 4) are potentially detectable by the consumer. The

393 differences between ceftiofur- and saline-treated as compared to tildipirosin- or tulathromycin-

394 darted animals (see Table 4) may not be detectable by the consumer even though statistically

395 significantly different.

397 Conclusions

Delivery of ceftiofur crystalline free acid, tildipirosin, and tulathromycin to cattle using pressureadjustable pneumatic gas-powered dart gun may result in pharmacokinetics and therefore meat withdrawal times and therapeutic efficacy that are different from those expected after conventional injection of these drugs. However, the most notable differences from published pharmacokinetic variable estimates as well as variability was with the ceftiofur crystalline free acid-treated animals. Delivery of tildipirosin and tulathromycin to cattle with dart gun may also result in detectable decreases in tenderness of harvested steaks. Delivery of ceftiofur crystalline free acid in this manner would be considered an illegal extralabel use and is therefore 406 discouraged.

\section{Acknowledgements}

409

The authors acknowledge Crystal Waters for the collection and analysis of tissue samples and initial tenderness data analysis, Cory Langston for discussions about the pharmacokinetic analysis, and Dan Hale and Davie Griffith for their assistance with study design and sample

412 preparation and handling. 
Coetzee JF, Kleinhenz MD, Magstadt DR, Cooper VL, Wulf LW, Van Engen NK, Smith JS, Rand N, KuKanich B, and Gorden PJ. 2018. Pneumatic dart delivery of tulathromycin in calves results in lower antimicrobial concentrations and increased biomarkers of stress and injection site inflammation compared with subcutaneous injection. J Anim Sci 96:3089-3101. 10.1093/jas/sky222

Cox SR, McLaughlin C, Fielder AE, Yancey MF, Bowersock TL, Garcia-Tapia D, Bryson L, Lucas MJ, Robinson JA, Nanjiani I, and Brown SA. 2010. Rapid and Prolonged Distribution of Tulathromycin into Lung Homogenate and Pulmonary Epithelial Lining Fluid of Holstein Calves Following a Single Subcutaneous Administration of $2.5 \mathrm{mg} / \mathrm{kg}$ Body Weight. International Journal of Applied Research in Veterinary Medicine 8:129137.

Falconer LL, and Anderson DP. 2006. The stocker cattle supply chain. Vet Clin North Am Food Anim Pract 22:297-303. 10.1016/j.cvfa.2006.03.003

Foster DM, Martin LG, and Papich MG. 2016. Comparison of Active Drug Concentrations in the Pulmonary Epithelial Lining Fluid and Interstitial Fluid of Calves Injected with Enrofloxacin, Florfenicol, Ceftiofur, or Tulathromycin. PLoS One 11:e0149100. 10.1371/journal.pone.0149100

George MH, Cowman GL, Tatum JD, and Smith GC. 1996. Incidence and sensory evaluation of injection-site lesions in beef top sirloin butts. J Anim Sci 74:2095-2103.

George MH, Morgan JB, Glock RD, Tatum JD, Schmidt GR, Sofos JN, Cowman GL, and Smith GC. 1995. Injection-site lesions: incidence, tissue histology, collagen concentration, and muscle tenderness in beef rounds. J Anim Sci 73:3510-3518.

Menge M, Rose M, Bohland C, Zschiesche E, Kilp S, Metz W, Allan M, Ropke R, and Nurnberger M. 2012. Pharmacokinetics of tildipirosin in bovine plasma, lung tissue, and bronchial fluid (from live, nonanesthetized cattle). J Vet Pharmacol Ther 35:550-559. 10.1111/j.1365-2885.2011.01349.x

Nowakowski MA, Inskeep PB, Risk JE, Skogerboe TL, Benchaoui HA, Meinert TR, Sherington J, and Sunderland SJ. 2004. Pharmacokinetics and lung tissue concentrations of tulathromycin, a new triamilide antibiotic, in cattle. Vet Ther 5:60-74.

Sullivan MM, VanOverbeke DL, Kinman LA, Krehbiel CR, Hilton GG, and Morgan JB. 2009. Comparison of the Biobullet versus traditional pharmaceutical injection techniques on injection-site tissue damage and tenderness in beef subprimals. J Anim Sci 87:716-722.

United States Food and Drug Administration Center for Veterinary Medicine. 2012. New Animal Drugs; Cephalosporin Drugs; Extralabel Animal Drug Use; Order of Prohibition. Federal Register 77:735-745.

United States Food and Drug Administration Center for Veterinary Medicine. 2020. Code of Federal Regulations; 21 CFR 522.313a; Implantation or Injectable Dosage Form New Animal Drugs; Ceftiofur crystalline free acid.

USDA. 2017. United States Standards for Grades of Carcass Beef. Available at https://www.ams.usda.gov/sites/default/files/media/CarcassBeefStandard.pdf(accessed February 2, 2020 2020).

Washburn K, Johnson R, Clarke CR, Anderson K, Lucas M, Bryson W, Robinson J, Dame K, Hubbard V, Callahan K, and Robb E. 2005. Penetration of ceftiofur into sterile vs. Mannheimia haemolytica-infected tissue chambers in beef calves after subcutaneous 
460

461

462

463

464

465

466

467

468

469

470

administration of ceftiofur crystalline free acid sterile suspension in the ear pinna. $J$ Vet Pharmacol Ther 28:247-251. 10.1111/j.1365-2885.2005.00642.x

Witte TS, Iwersen M, Kaufmann T, Scherpenisse P, Bergwerff AA, and Heuwieser W. 2011. Determination of ceftiofur derivatives in serum, endometrial tissue, and lochia in puerperal dairy cows after subcutaneous administration of ceftiofur crystalline free acid. $J$ Dairy Sci 94:284-290. 10.3168/jds.2010-3645

Woodrow JS, Caldwell M, Cox S, Hines M, and Credille BC. 2016. Comparative plasma pharmacokinetics of ceftiofur sodium and ceftiofur crystalline-free acid in neonatal calves. J Vet Pharmacol Ther 39:271-276. 10.1111/jvp. 12275 
Figure 1

Mean serum concentrations of ceftiofur after single dose of $6.6 \mathrm{mg} / \mathrm{kg}$ via dart (see text for explanation of dosing) in 8 animals

Error bars are standard deviation.

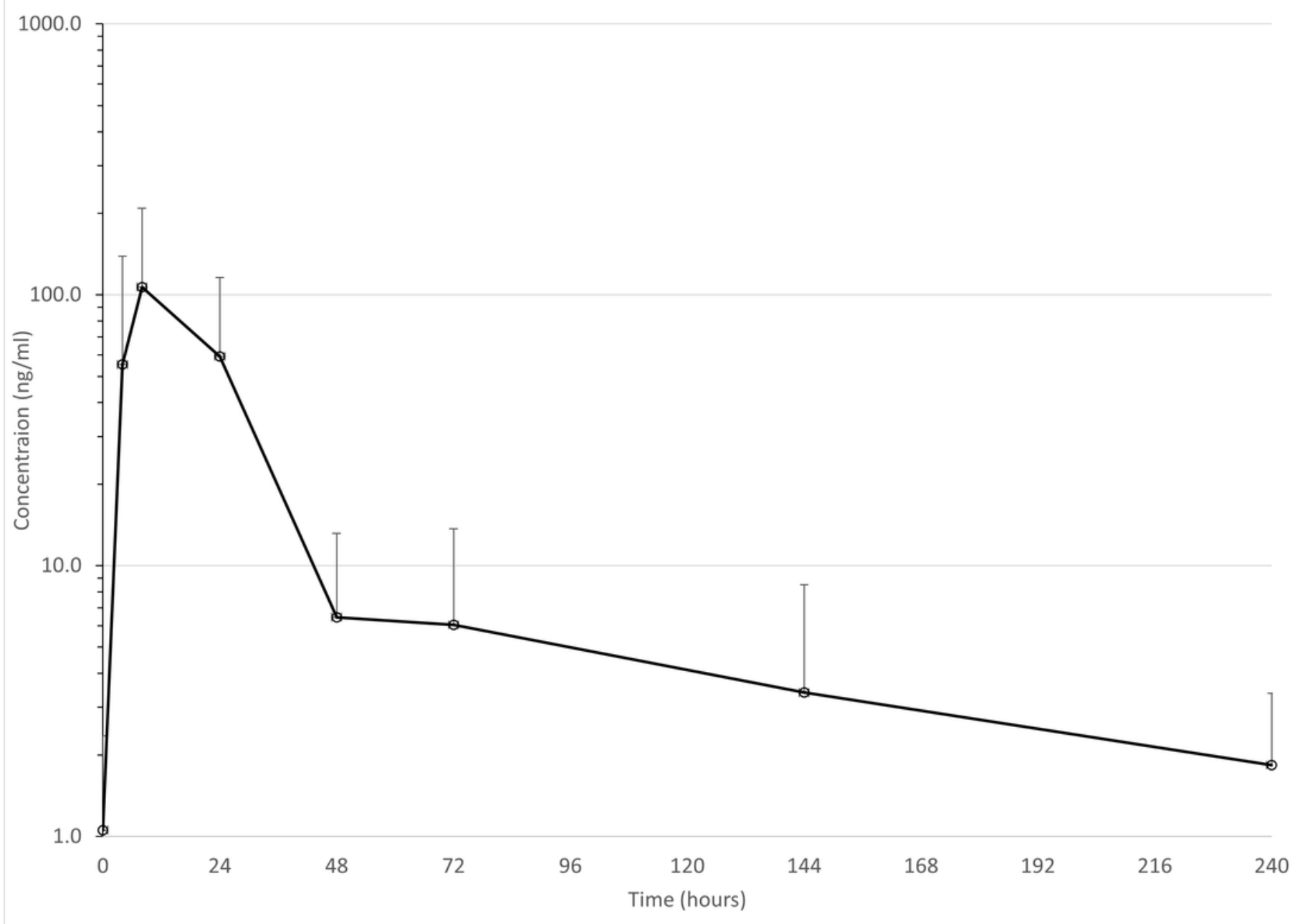


Figure 2

Mean serum concentrations of tildopirosin after single dose of $4 \mathrm{mg} / \mathrm{kg}$ via dart (see text for explanation of dosing) in 6 animals

Error bars are standard deviation.

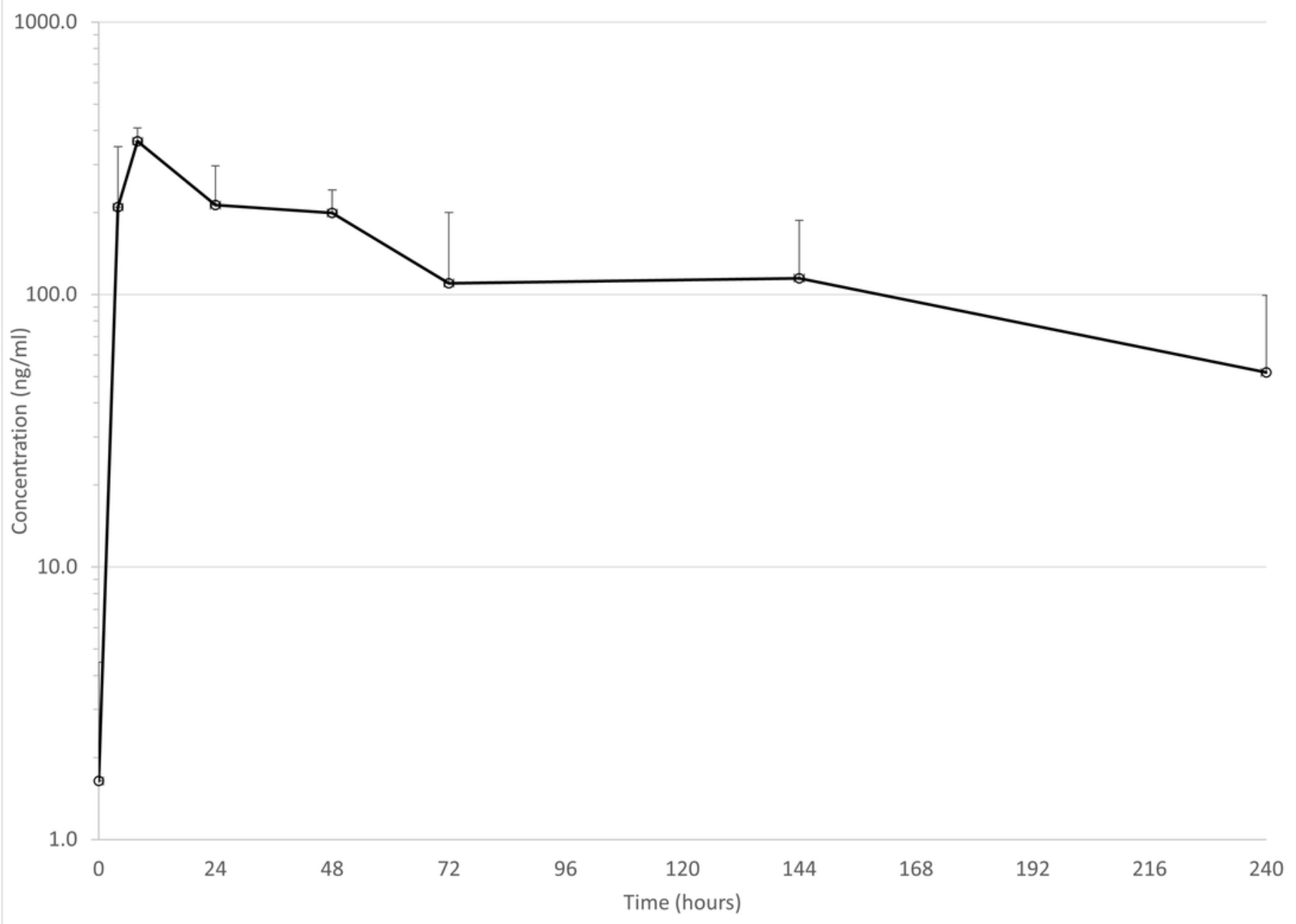


Figure 3

Mean serum concentrations of tulathromycin after single dose of $2.5 \mathrm{mg} / \mathrm{kg}$ via dart (see text for explanation of dosing) in 6 animals

Error bars are standard deviation.

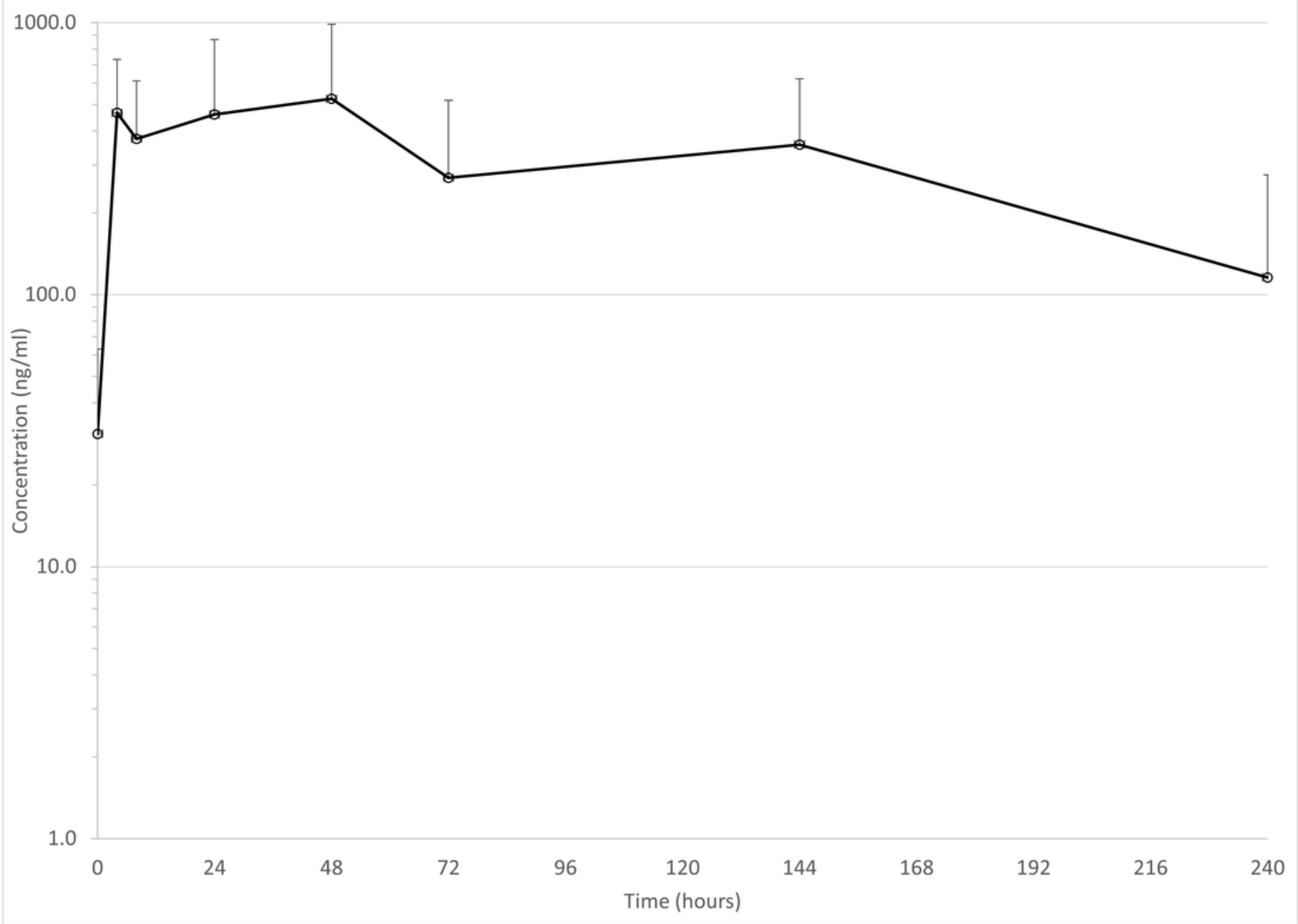




\section{Table $\mathbf{1}$ (on next page)}

Estimated pharmacokinetic parameters for ceftiofur after single dose of $6.6 \mathrm{mg} / \mathrm{kg}$ ceftiofur crystalline free acid via dart

See Materials and Methods for explanation of darting. 
Table 1

Estimated pharmacokinetic parameters for ceftiofur after single dose of $6.6 \mathrm{mg} / \mathrm{kg}$ ceftiofur crystalline free acid via dart (see Materials and Methods for explanation of darting)

\begin{tabular}{|c|c|c|c|c|c|c|c|c|c|c|c|c|}
\hline Animal No. & & 599 & 608 & 638 & 645 & 680 & 1 & 2 & 3 & Mean & $S D$ & Median \\
\hline $\mathbf{C}_{\max }$ & $\mathrm{ng} / \mathrm{ml}$ & 155 & 16 & 241 & 238 & 174 & 134 & 17 & 41 & 127 & 93 & 145 \\
\hline $\mathbf{T}_{\max }$ & $\mathrm{hr}$ & 8 & 8 & 4 & 8 & 8 & 24 & 24 & 24 & $*$ & & 8 \\
\hline$\lambda \mathbf{z}$ & $/ \mathrm{hr}$ & 0.0057 & 0.0051 & 0.0226 & 0.0108 & 0.0091 & 0.0163 & 0.0211 & 0.0081 & $\mathbf{0}$ & 0 & 0 \\
\hline$t_{1 / 2 \lambda z}$ & $\mathrm{hr}$ & 123 & 136 & 31 & 64 & 76 & 42 & 33 & 85 & 74 & 40 & 70 \\
\hline $\mathbf{A} \mathbf{U C}_{\text {0-obs }}$ & $\mathrm{ng} * \mathrm{hr} / \mathrm{ml}$ & 4096 & 898 & 6854 & 4161 & 2645 & 4783 & 704 & 2158 & 3287 & 2085 & 3371 \\
\hline $\begin{array}{l}\mathrm{AUC}_{0 \text {-inf }} \\
\mathrm{AUC} \%\end{array}$ & $\mathrm{ng} * \mathrm{hr} / \mathrm{ml}$ & 4542 & 1036 & 6892 & 4336 & 2753 & 4960 & 746 & 2749 & 3502 & 2077 & 3544 \\
\hline Extrap & & 10 & 13 & 1 & 4 & 4 & 4 & 6 & 21 & 8 & 7 & 5 \\
\hline $\mathbf{A} \mathbf{U} \mathbf{M C}_{0-\mathrm{obs}}$ & $\mathrm{ng}^{2 *} \mathrm{hr} / \mathrm{ml}$ & 258400 & 76492 & 137545 & 83398 & 79881 & 221486 & 31720 & 131242 & 127520 & 77525 & 107320 \\
\hline $\mathbf{A U M C} \mathbf{C}_{0 \text {-inf }}$ & $\mathrm{ng}^{2 *} \mathrm{hr} / \mathrm{ml}$ & 444331 & 136637 & 148474 & 141868 & 117522 & 274959 & 39879 & 345440 & 206139 & 135572 & 145171 \\
\hline MRT $_{\text {0-obs }}$ & $\mathrm{hr}$ & 63 & 85 & 20 & 20 & 30 & 46 & 45 & 61 & 46 & 23 & 46 \\
\hline MRT $_{0 \text {-inf }}$ & $\mathrm{hr}$ & 98 & 132 & 22 & 33 & 43 & 55 & 53 & 126 & 70 & 43 & 54 \\
\hline
\end{tabular}

*Median is preferred for this parameter, so mean is not reported.

$\mathrm{T}_{\max }=$ time of peak serum drug concentration; $\mathrm{C}_{\max }=$ peak drug concentration; $\mathrm{t}_{1 / 2}=$ apparent elimination half-life, calculated as

$\ln (2) / \lambda_{z}, \lambda_{z}$ being the first order rate constant associated with the terminal portion of the time-concentration curve as estimated by

linear regression of time vs. $\log$ concentration; $\mathrm{AUC}_{0 \text {-obs }}=$ area under the time-concentration curve from time zero to last observed

concentration calculated by the linear trapezoidal rule; $\mathrm{AUC}_{0 \text {-inf }}=$ area under the time-concentration curve from time zero extrapolated

to infinity, calculated by adding the last observed concentration divided by $\lambda_{\mathrm{z}}$ to the $\mathrm{AUC}_{0 \text {-obs}} ; \mathrm{AUMC}_{0 \text {-obs }}=$ area under the moment

curve from time zero to last observed concentration; $\mathrm{AUMC}_{0 \text {-inf }}=$ area under the moment curve from time zero extrapolated to 
infinity; $\mathrm{MRT}_{0 \text {-obs }}=$ mean resident time estimated using time zero to last observed concentrations, calculated as $\mathrm{AUMC}_{0 \text {-obs }} / \mathrm{AUC}_{0 \text {-obs; }}$

$15 \mathrm{MRT}_{0 \text {-inf }}=$ mean residence time estimated using time zero to infinity, calculated as $\mathrm{AUMC}_{0 \text {-inf }} / \mathrm{AUC}_{0 \text {-inf }}$ 


\section{Table 2 (on next page)}

Estimated pharmacokinetic parameters for tildopirosin after single dose of $4 \mathrm{mg} / \mathrm{kg}$ via dart

(see text for explanation of dosing) 
1 Table 2

2 Estimated pharmacokinetic parameters for tildopirosin after single dose of $4 \mathrm{mg} / \mathrm{kg}$ via dart (see Materials and Methods for explanation of darting)

4

\begin{tabular}{|c|c|c|c|c|c|c|c|c|c|c|}
\hline Animal No. & & 555 & 582 & 594 & 634 & 640 & 682 & Mean & $S D$ & Median \\
\hline $\mathbf{C}_{\max }$ & $\mathrm{ng} / \mathrm{ml}$ & 366 & 310 & 354 & 346 & 424 & 405 & 368 & 41 & 360 \\
\hline $\mathbf{T}_{\max }$ & $\mathrm{hr}$ & 8 & 8 & 8 & 4 & 8 & 8 & 7 & 2 & 8 \\
\hline$\lambda \mathbf{z}$ & $/ \mathrm{hr}$ & 0.0073 & 0.0075 & 0.0108 & 0.0099 & 0.0105 & 0.0026 & 0.0081 & 0.0031 & 0 \\
\hline $\mathbf{t}_{1 / 2 \lambda \mathbf{z}}$ & $\mathrm{hr}$ & 95 & 92 & 64 & 70 & 66 & 267 & 109 & 79 & 81 \\
\hline $\mathbf{A U C} \mathbf{C}_{0-\mathrm{obs}}$ & $\mathrm{ng}{ }^{*} \mathrm{hr} / \mathrm{ml}$ & 19326 & 37150 & 26495 & 13112 & 45286 & 44372 & 30957 & 13396 & 31823 \\
\hline $\mathbf{A U} \mathbf{C}_{0 \text {-inf }}$ & $\mathrm{ng} * \mathrm{hr} / \mathrm{ml}$ & 25433 & 43122 & 28009 & 14725 & 49426 & 100368 & 43514 & 30540 & 35566 \\
\hline AUC \% Extrap & & 24 & 14 & 5 & 11 & 8 & 56 & 20 & 19 & 12 \\
\hline $\mathbf{A} \mathbf{U} \mathbf{M C}_{\mathbf{0 - o b s}}$ & $\mathrm{ng}^{2 *} \mathrm{hr} / \mathrm{ml}$ & 1324693 & 3596137 & 2049570 & 818095 & 3936357 & 4662887 & 2731290 & 1551437 & 2822854 \\
\hline $\mathbf{A U M C}_{0 \text {-inf }}$ & $\mathrm{ng}^{2 *} \mathrm{hr} / \mathrm{ml}$ & 3623687 & 5824633 & 2553052 & 1368295 & 5325416 & 39711236 & 9734386 & 14779989 & 4474552 \\
\hline MRT $_{0-\text { obs }}$ & $\mathrm{hr}$ & 69 & 97 & 77 & 62 & 87 & 105 & 83 & 16 & 82 \\
\hline MRT $_{\text {o-inf }}$ & $\mathrm{hr}$ & 142 & 135 & 91 & 93 & 108 & 396 & 161 & 117 & 121 \\
\hline
\end{tabular}

6 See Table 1 for abbreviations 


\section{Table $\mathbf{3}$ (on next page)}

Estimated pharmacokinetic parameters for tulathromycin after single dose of $2.5 \mathrm{mg} / \mathrm{kg}$ via dart

(see text for explanation of dosing) 
1 Table 3

2 Estimated pharmacokinetic parameters for tulathromycin after single dose of $2.5 \mathrm{mg} / \mathrm{kg}$ via dart (see Materials and Methods for explanation of darting)

\begin{tabular}{|c|c|c|c|c|c|c|c|c|c|c|}
\hline Animal No. & & 569 & 637 & 646 & 670 & 671 & 672 & Mean & $S D$ & Median \\
\hline $\mathrm{C}_{\max }$ & $\mathrm{ng} / \mathrm{ml}$ & 544 & 797 & 618 & 1221 & 421 & 109 & 619 & 374 & 581 \\
\hline $\mathbf{T}_{\max }$ & $\mathrm{hr}$ & 4 & 4 & 48 & 24 & 8 & 8 & 16 & 17 & 8 \\
\hline$\lambda z$ & $/ \mathrm{hr}$ & 0.0109 & 0.0062 & 0.0081 & 0.0042 & 0.0070 & 0.0058 & 0.0070 & 0.0023 & 0 \\
\hline $\mathbf{t}_{1 / 2 \lambda z}$ & $\mathrm{hr}$ & 63 & 111 & 86 & 166 & 99 & 118 & 107 & 35 & 105 \\
\hline$A U C_{110-o b s}$ & $\mathrm{ng} * \mathrm{hr} / \mathrm{ml}$ & 41330 & 71143 & 89334 & 186274 & 52928 & 7906 & 74819 & 61196 & 62036 \\
\hline $\mathbf{A U} \mathbf{C}_{0 \text {-inf }}$ & $\mathrm{ng}^{*} \mathrm{hr} / \mathrm{ml}$ & 43032 & 81506 & 101740 & 290168 & 61856 & 10282 & 98097 & 99234 & 71681 \\
\hline AUC \% Extrap & & 4 & 13 & 12 & 36 & 14 & 23 & 17 & 11 & 14 \\
\hline $\mathrm{AUMC}_{0-\mathrm{obs}}$ & $\mathrm{ng}^{2 *} \mathrm{hr} / \mathrm{ml}$ & 3709741 & 6992264 & 8739931 & 19268859 & 4903251 & 677774 & 7381970 & 6447784 & 5947758 \\
\hline $\mathrm{AUMC}_{0 \text {-inf }}$ & $\mathrm{ng}^{2 *} \mathrm{hr} / \mathrm{ml}$ & 4273912 & 11144018 & 13248509 & 69034189 & 8323160 & 1654218 & 17946334 & 25390091 & 9733589 \\
\hline MRT $_{0 \text {-obs }}$ & $\mathrm{hr}$ & 90 & 98 & 98 & 103 & 93 & 86 & 95 & 6 & 95 \\
\hline MRT $_{\text {0-inf }}$ & $\mathrm{hr}$ & 99 & 137 & 130 & 238 & 135 & 161 & 150 & 47 & 136 \\
\hline
\end{tabular}

6 See Table 1 for abbreviations 


\section{Table 4 (on next page)}

Least squares means for Warner-Bratzler Shear Force $(\mathrm{kg})$ for top loin and bottom round steaks 
1 Table 4. Least squares means for Warner-Bratzler Shear Force (kg) for top loin and bottom round steaks. 4 5 6

7

10

11

12

13

14

15

16

17

18

19

20

21

22

23

24

25

26

27

28

29

30

31

32

33

Top Loin Steaks $\quad$ Bottom Round Steaks

Treatment

Top Loin Steaks $\quad$ Bottom Round Steaks

Bottom Round Steaks

Dartedle $^{\mathrm{e}} \quad$ Control $^{\mathrm{e}}$

\begin{tabular}{|c|c|c|c|c|}
\hline Dart Treatment & $0.21^{\mathrm{d}}$ & $0.003^{\mathrm{d}}$ & $0.08^{\mathrm{d}}$ & \\
\hline CCFA & 3.04 & $3.10^{\mathrm{a}}$ & $3.22^{\mathrm{ab}}$ & $3.10^{\mathrm{a}}$ \\
\hline SALN & 2.77 & $3.11^{\mathrm{a}}$ & $3.27^{\mathrm{ab}}$ & $3.11^{\mathrm{ab}}$ \\
\hline TILD & 3.19 & $3.44^{\mathrm{b}}$ & $3.71^{\mathrm{b}}$ & $3.08^{\mathrm{a}}$ \\
\hline TULA & 3.00 & $3.45^{b}$ & $3.51^{\mathrm{ab}}$ & $3.32^{\mathrm{ab}}$ \\
\hline Position & $0.73^{d}$ & $0.48^{\mathrm{d}}$ & & \\
\hline$\overline{1}$ & 3.05 & 3.43 & & \\
\hline 2 & 3.06 & 3.27 & & \\
\hline 3 & 3.02 & 3.16 & & \\
\hline 4 & 2.86 & 3.36 & & \\
\hline 5 & & 3.28 & & \\
\hline 6 & & 3.15 & & \\
\hline Age Day & $<0.001^{\mathrm{d}}$ & & & \\
\hline 1 & $3.85^{\mathrm{c}}$ & & & \\
\hline 7 & $3.11^{b}$ & & & \\
\hline 14 & $2.67^{\mathrm{ab}}$ & & & \\
\hline 21 & $2.36^{\mathrm{a}}$ & & & \\
\hline Root Mean Square Error & 0.838 & 0.693 & 0.672 & \\
\hline
\end{tabular}

abcMean values within a column and effect followed by the same letter are not significantly different $(\mathrm{P}>0.05)$.

dP value for the treatment effect from the Analysis of Variance table.

${ }^{\mathrm{e}}$ Control = right side, not darted; Darted = left side, darted from the steak removed where the dart insertion point was identified.

Positions in top loins steaks (see text for details): $1=$ steak from anterior edge of Longissimus dorsi lumborum muscle; $2=$ steak immediately posterior to $1 ; 3=$ steak immediately posterior to 2 ; 4=steak immediately posterior to 3

Positions in bottom round steaks (see text for details): 1=steak from presumed site of dart entry, $2=1^{\text {st }}$ steak distal to presumed site of dart entry, $3=2^{\text {nd }}$ steak distal to presumed site of dart entry, $4=1^{\text {st }}$ steak proximal to presume site of dart entry, $5=2^{\text {nd }}$ steak distal to presumed site of dart entry, $6=$ steak from non-darted (right) side at similar location to the darted side 


\section{Table 5 (on next page)}

Least squares means for carcass quality and yield factors by dart treatment 
1 Table 4. Least squares means for carcass quality and yield factors by dart treatment.

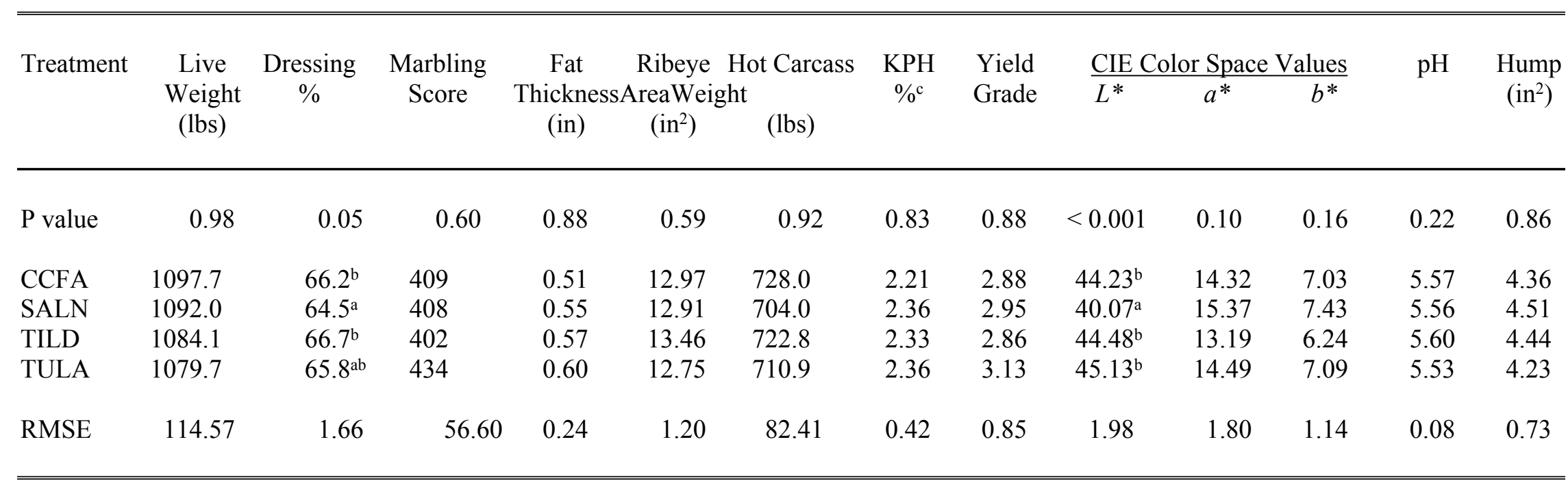

${ }^{\mathrm{ab}}$ Mean values within a column and effect followed by the same letter are not significantly different (P $\left.>0.05\right)$.

${ }^{\mathrm{c}} \mathrm{KPH} \%=$ Kidney, Pelvic and Heart Fat 